\title{
Indoor Characterization of Power Delay Profile Using Equivalent Antenna Representation in Uniform FDTD Lattices
}

\author{
Bruno W. Martins, Rodrigo M. S. de Oliveira, Victor Dmitriev, \\ Fabrício J. B. Barros and Nilton R. N. M. Rodrigues. \\ Federal University of Pará, Belém-PA, Brazil \\ e-mails:brunow@ufpa.br,rmso@ufpa.br,victor@ufpa.br,fbarros@ufpa.br and niltonrodolfo@hotmail.com
}

\begin{abstract}
It is shown that proper representation of transmitting and receiving antennas in indoor environments are decisive for accurate prediction of electromagnetic power transfer parameters using the standard 3D-FDTD method. With the standard Yee method, it is not always possible to precisely describe all the geometrical details of electrically small antennas. A new approach for description of the discone antenna in uniform 3D-FDTD lattice is presented. The calculations are validated by comparing FDTD results with the polyhedral beam-tracing method and experimental data.
\end{abstract}

Index Terms - Channel characterization, power delay profile, 3D-FDTD, electrically small antennas, large computational environments.

\section{INTRODUCTION}

For designing communication systems, the electromagnetic characteristics of communication channel must be trustworthily known [1]. With computational simulations [2], it is possible, for example, to determine the optimal position of the transmitting antenna to maximize the coverage area and to identify multipath phenomena calculating the power delay profile (PDP). This parameter can be calculated using the procedure given in $[1,3]$, which avoids the use of deconvolution algorithms to obtain impulsive responses of the channel.

Based on the discone antenna in [4] which is electrically small, a new antenna was designed, built and used in the measurements presented in [3,5]. Its straightforward numerical representation in such electrically large indoor environments is limited by computational restrictions when the standard 3D finite-difference time-domain (3D-FDTD) method [2] is used, even when a powerful computer cluster is used. It means that an extremely high number of Yee cells [2,6] would be required for modeling the entire environment containing the antenna if a globally uniform grid is used. Alternative solutions would be using locally non-orthogonal or locally refined orthogonal grids $[2,6]$, which can be, respectively, mathematically intricate [7] or numerically demanding because stability is usually achieved using considerably small time steps [2].

In this paper we introduce an equivalent FDTD representation of the discone antenna, which is used as transmitter and receiver, in such a way that most of its characteristics (polarization, operating frequency band and radiation patterns) are approximated using a globally coarse grid, thus preserving 
the simplicity, elegance and the original efficiency of Yee method. In addition, the introduced procedure significantly simplifies computer implementation because complications inherent to nonuniform or non-orthogonal grids are circumvented $[2,6]$. With the proposed approach, the obtained numerical results characterizing the indoor environment become much closer to the experimental data than the numerical results presented earlier in [3].

\section{MEASUREMENT ENVIRONMENT}

\section{A. Indoor environment}

In Fig. 1, we show the indoor environment used for performing experimental and numerical analyses of time spread parameters of electromagnetic signals. It is a corridor on the second floor of the building Cardeal LEME at PUC-Rio university. During experiments, a vector network analyzer was used as a transceiver exciting 1601 sinusoidal carriers uniformly separated by approximately 0.53 $\mathrm{MHz}$ in the frequency band ranging from $950 \mathrm{MHz}$ to $1800 \mathrm{MHz}$, as presented in [3,5]. A descriptive list of the parameters of the measurements is given in Table I.

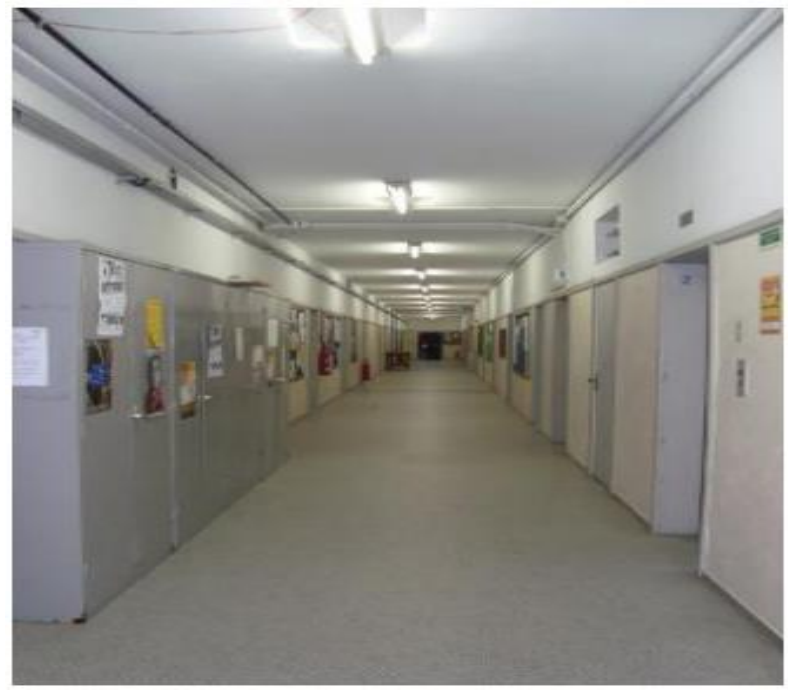

Fig. 1. Corridor of field measurements: this environment is reproduced numerically using the 3D-FDTD Method.

TABLE I. MEASUREMENT SETUP PARAMETERS [3,5]

\begin{tabular}{ll}
\hline \multicolumn{1}{c}{ Parameters } & \multicolumn{1}{c}{ Values } \\
\hline Frequency range & 950 to $1800 \mathrm{MHz}$ \\
Bandwidth & $850 \mathrm{MHz}$ \\
Number of samples & 1601 \\
Time resolution $(\Delta \tau)$ & $1.17 \mathrm{~ns}$ \\
Maximum delay & $1882.35 \mathrm{~ns}$ \\
Analysis time & $346 \mathrm{~ns}$ \\
Frequency spacing between samples & $0.53 \mathrm{MHz}$ \\
Transmission power & $10 \mathrm{dBm}$ \\
Amplifier gain & $25 \mathrm{~dB}$ \\
Cable and connector losses & $5 \mathrm{~dB}$ \\
\hline
\end{tabular}

Fig. 2 shows the floor plan of the indoor environment of Fig. 1. During measurements, there were no people in the environment, excepting two engineers performing the measurements. The 
transmitting and receiving antennas have been positioned at the height of $1.5 \mathrm{~m}$ above the floor for performing LOS (line-of-sight) propagation analysis. The transmitter was positioned at 88 different points, being moved away from the receiver $0.5 \mathrm{~m}$ each time in straight line in the corridor, as indicated by Fig. 2. The first position of the transmitter $\mathrm{Tx}_{1}$ is one meter away from the receiver $\mathrm{Rx}$ and the last position analyzed $\mathrm{Tx}_{88}$ is $44.5 \mathrm{~m}$ away from $\mathrm{Rx}$. Notice that the used time to obtain the amplitudes and phases is $1.882 \mu$ s for each position of the transmitter.

\section{B. Numerical modeling of environment}

In our computer representation of the environment defined by the floor plan in Fig. 2, most objects were modelled for signal prediction. Electrically small objects, as the fire extinguisher and the coffee machine, are also considered in our numerical FDTD model. In Table II, the dimensions and electromagnetic characteristics (relative permittivity $\varepsilon_{r}$ and conductivity $\sigma$ ) of each object considered in the simulation can be seen. The FDTD representation of the environment was developed using the software SAGS [8]. The polyhedral beam-tracing model follows [3,9]. For the FDTD model, the parameters in Table II were set individually for each field component in Yee's cells to properly define each dielectric or conducting block forming the indoor scenario. The discretization is uniform throughout the environment and it is based on cubic Yee cells with $3 \mathrm{~cm}$-long edges $(\Delta=3 \mathrm{~cm})$. The total FDTD mesh size is $3120 \times 520 \times 175$ and the time step was set to $99 \%$ of Courant's limit [2]. FDTD and PBT models were applied to the environment shown in Fig. 2 with the parameters of Table II.

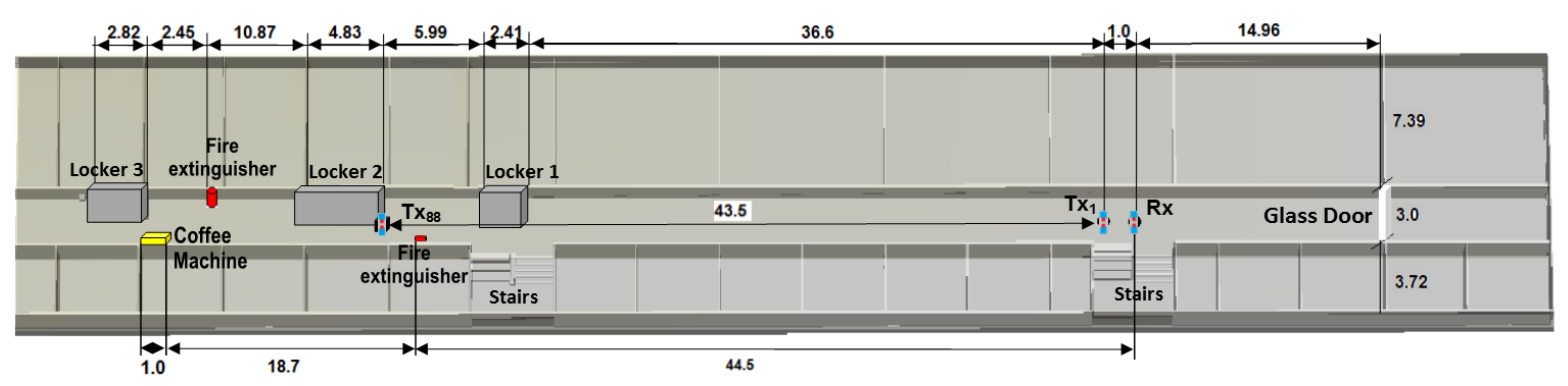

Fig. 2 SAGS 3D-view of the FDTD model of LEME, with position markers of transmitter $\left(\mathrm{Tx}_{1}\right.$ and $\left.\mathrm{Tx}_{88}\right)$ and receiver $(\mathrm{Rx})$; all dimensions are given in meters.

TABLE II. PHYSICAL PARAMETERS OF OBJECTS CONSIDERED IN FDTD AND PBT MODELS

\begin{tabular}{cccccc}
\hline Object & Material & Dimensions $(\mathbf{m})$ & $\boldsymbol{\varepsilon}_{\boldsymbol{r}}$ & $\boldsymbol{\sigma}(\mathbf{S} / \mathbf{m})$ & $\boldsymbol{\mu}_{\boldsymbol{r}}$ \\
\hline Walls & Brick & thickness: 0.15 & 4 & 0.0016 & 1 \\
Floor/stairs & Concrete & thickness: 0.30 & 5 & 0.011 & 1 \\
Ceiling & plaster & thickness: 0.03 & 2.8 & 0.0153 & 1 \\
Locker & metal & $2.1 \times 3.0 \times 1.0$ & 1 & $1 \times 10^{9}$ & 1 \\
\hline
\end{tabular}




\section{THE PROPOSED EQUIVALENT FDTD REPRESENTATION OF THE DISCONE ANTENNA}

The antenna used in the measurements is a discone antenna illustrated by Figs. 3(a) and 3(b). For the frequency range 950-1800 MHz, its gain as a function of the elevation angle $\theta$ is shown in Fig.4. The return loss of the discone antenna is approximately constant under $-15 \mathrm{~dB}$, as shown by Fig. 5 . The discone antenna has an omnidirectional radiation pattern and return loss below $-15 \mathrm{~dB}$ in frequency range of interest, as shown by Figs. 4 and 5, respectively.

As previously discussed, there are issues for representing computationally the discone antenna geometry of Fig.3(a) using the standard 3D-FDTD method for the electrically large problem. Thus, an equivalent representation of the antenna is suggested for modeling the device in uniform FDTD lattices (Fig. 3c).

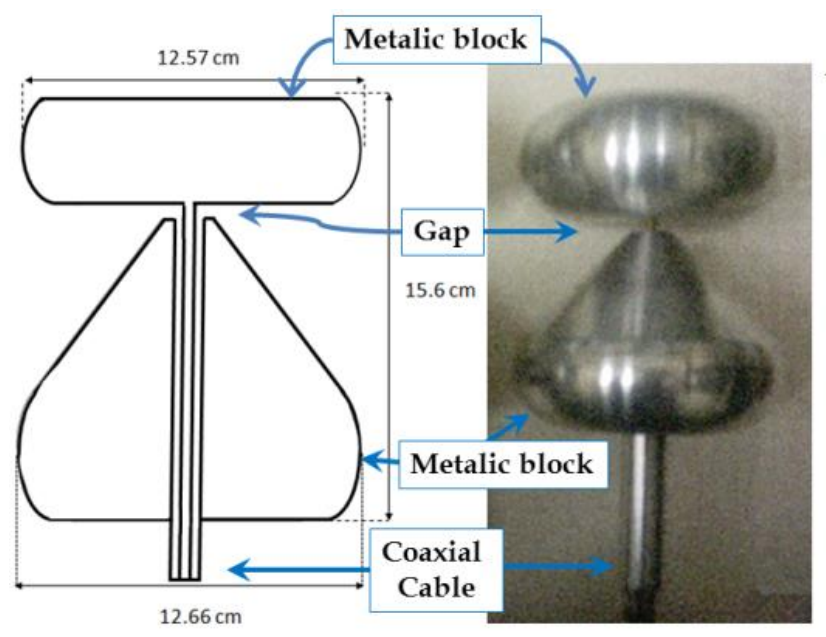

(a)

(b)

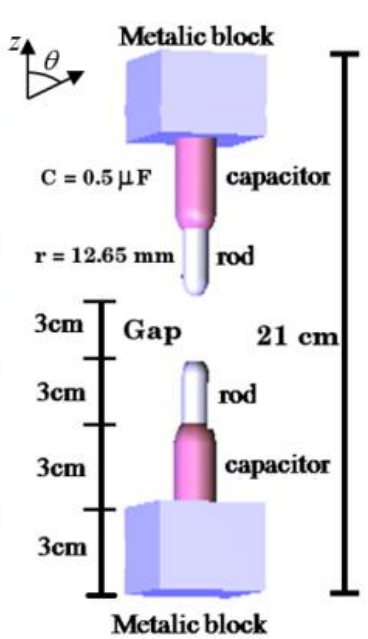

(c)

Fig. 3. Discone antenna: (a) schematics, (b) photograph of the constructed antenna used in experiments and (c) optimized equivalent antenna for FDTD simulations.

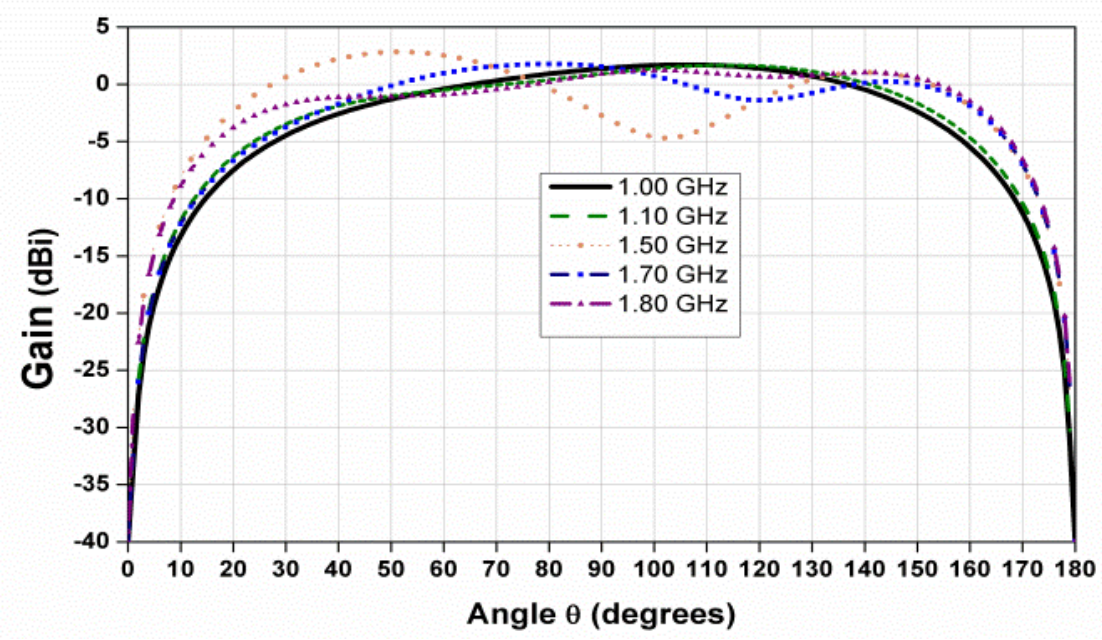

Fig. 4. The Gain of the discone antenna used in PBT model of [9], calculated for several frequencies using method of moments (MoM). 


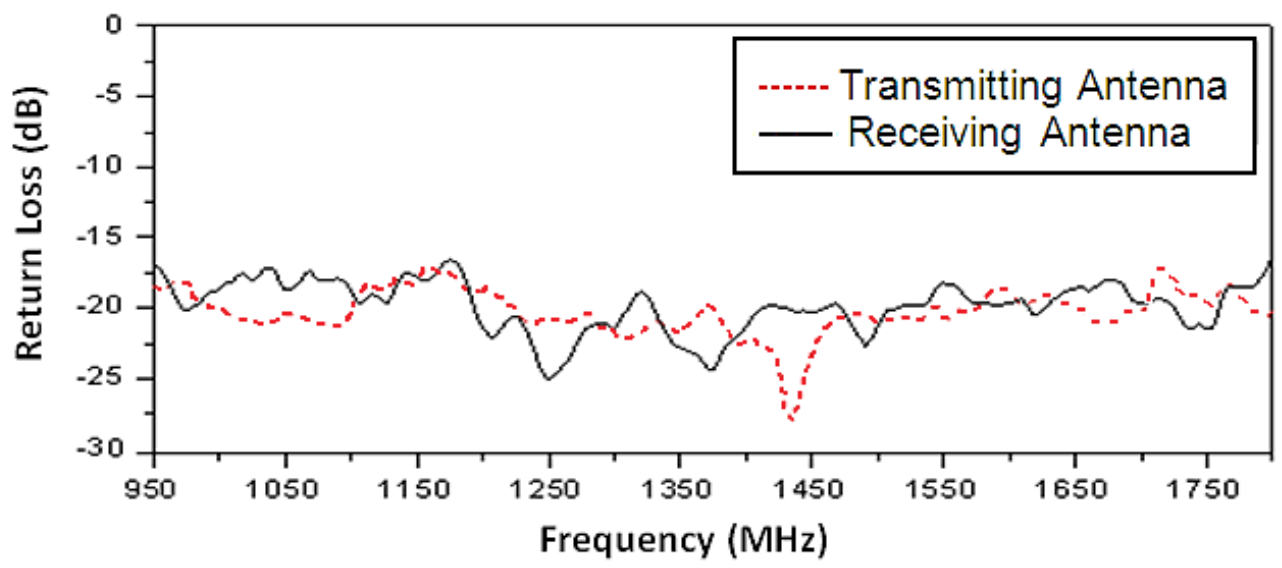

Fig. 5. Measured return losses of discone antennas.

For the maximum frequency considered $(1.8 \mathrm{GHz}$, Fig.5), we have a wavelength of approximately $16.66 \mathrm{~cm}$. We see in Fig. 3 that the total height of the antenna is $15.6 \mathrm{~cm}$, which is smaller than the minimum wavelength considered. All other dimensions of geometric details are even smaller. For the lower frequencies considered (Fig. 5), the electric lengths of the constituent parts of the discone antenna are even less significant. Due to the large electrical length of the indoor environment, we have used $\Delta=3 \mathrm{~cm}$. Local refinements of FDTD discretization over the antenna could be used for representing the small features of the device, but this would force substantial reduction of the time step $\Delta \mathrm{t}$ (which must be avoided) [2]. Although the minor geometric details are not extremely important (for the considered electric lengths), larger details must be geometrically approximated in order to produce properly approximated radiation patterns. That is why the proposed model has the shape shown in Fig.3(c). However, because $\Delta=3 \mathrm{~cm}$, this representation is larger than the original antenna. Thus, in order to reduce its electric length, capacitors have been used for compensating for the reactive impedance, which is inductive. Clearly, the antenna polarization is preserved in the equivalent model.

To compare the equivalent representation and the real discone antenna, we have calculated the return loss $R L$ given by

$$
R L=-20 \cdot \log _{10}\left|\frac{Z-Z_{S}}{Z+Z_{S}}\right|
$$

In (1), $Z_{S}$ is the load impedance ( $\left.50 \Omega\right)$ and $Z$ is the impedance of the simulated antenna obtained through point-by-point division of the Fourier transforms of voltage $V(t)$ and current $I(t)$ in a discrete frequency spectrum. Mathematically, one has

$$
Z(f)=\frac{\mathcal{F}\{V(t)\}}{\mathcal{F}\{I(t)\}}
$$

The voltage $V(t)$ is the excitation pulse, which is a function of time $t$ (Fig. 6(a)). The voltage is imposed by exciting the $z$-component of electric field between the terminals (in the gap) of the 
antenna (Fig. 3(c)). The source is implemented by setting $E_{z}^{\text {source }}=V(t) / \Delta$, where $\Delta$ is the FDTD spatial increment (cubic Yee cell edge length). The pulse $V(t)$ is a Gaussian monocycle function (time derivative of a Gaussian pulse) given by

$$
V(t)=A_{p}\left(\frac{t-3.0 T}{T}\right) \exp \left[-\left(\frac{t-3.0 T}{T}\right)^{2}\right]
$$

where $A_{p}=1$ and $T=6.53846 \times 10^{-10} \mathrm{~s}$ are the pulse amplitude and width, respectively. As it can be seen in Fig. 6(a), the pulse has duration of approximately $0.85 \mathrm{~ns}$. The frequency range of the excitation signal in Fig. 6(b) contains significant power in appropriate frequency band between 950 MHz and $1800 \mathrm{MHz}$. The current is calculated in one of the antenna rods, in the cell neighboring the electric field excitation cell (antenna's gap), by evaluating the curl of magnetic field around the cylindrical rod, which is multiplied by the area of the face of secondary Yee's cell [2] orthogonal to the referred rod.

With this data, it is possible to determine the return loss of the preliminary dipole antenna. The antenna return loss is below $-2 \mathrm{~dB}$ (black solid line) in the desired frequency range, as shown by Fig. 7(a). This value is above the value produced and measured for the real antenna. Thus, adjustments for this preliminary antenna are important for providing a return loss profile similar to those shown by Fig. 5.

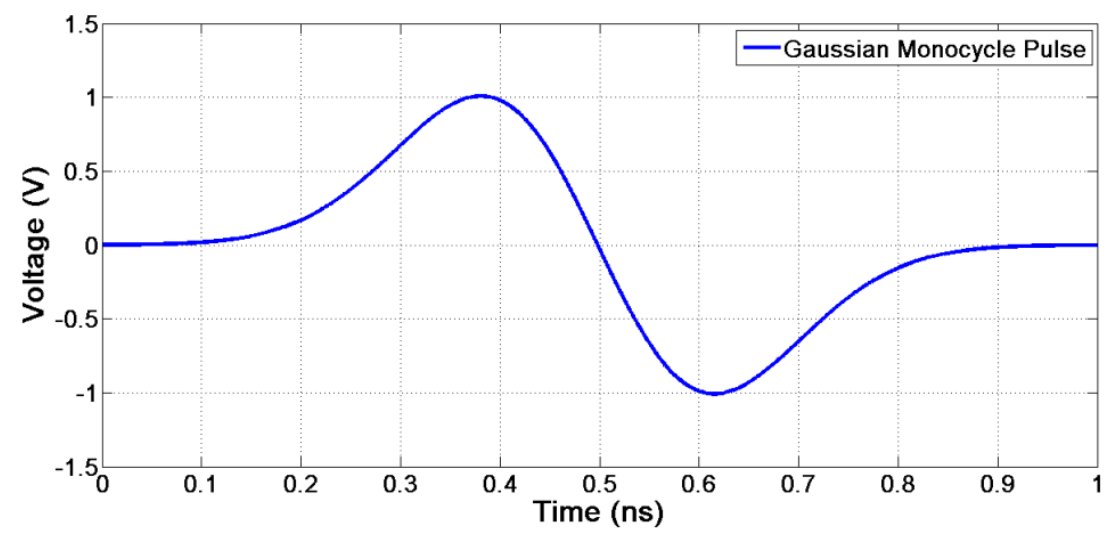

(a)

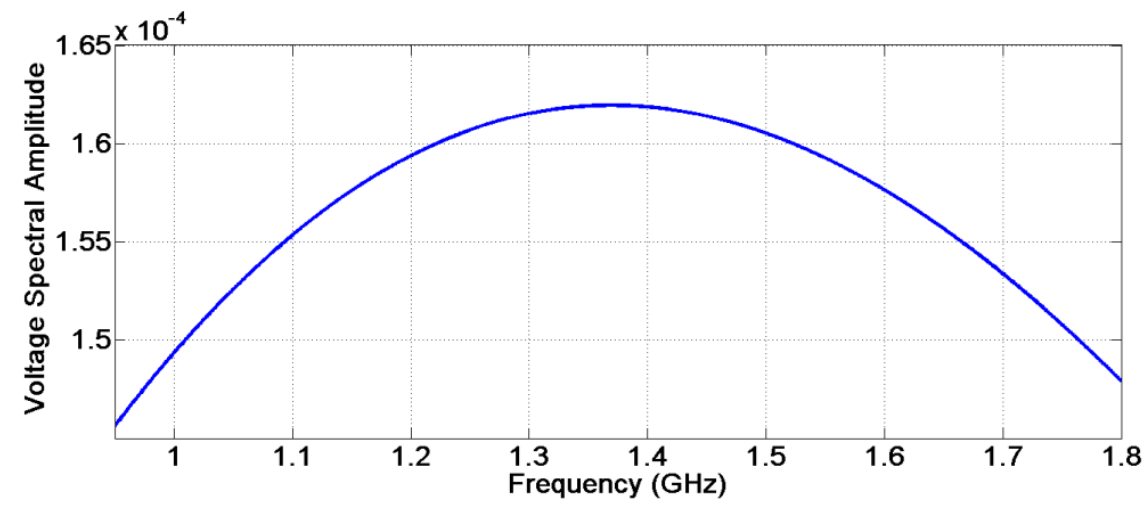

(b)

Fig. 6. Voltage across terminals of modeled transmitting antenna: (a) time domain and (b) frequency domain. 
It is known, from physics, that reducing the length of the antenna would displace the return loss point of minimum to a higher frequency, as required. However, this adjustment would also lead to very small Yee cells in classic FDTD method, increasing computational demands. Furthermore, local grid refinements (around the antennas) would impose the use of exceedingly small time steps [2].

Numerous tests were conducted considering adjustments to the model proposed. The goals are to make the simulated antenna to provide a return loss profile under $-4 \mathrm{~dB}$ or $-6 \mathrm{~dB}$ (as it is expected for indoor applications [10-13] and to approximate the gain of the equivalent antenna to the gain of the antenna used in experiments. For this purpose, the model shown in Fig. 3(c) is proposed. For representing the metallic parts indicated in Figs. 3(a) and 3(b), we have included cubic metallic blocks in the equivalent model illustrated by Fig. 3(c). Two syntonizing capacitors, modelled using the technique described in [14], are incorporated between the rods and the metallic blocks, as shown by Fig. 3 (c). The idea is to tune the antenna with the band of interest, i.e., to provide the impedance matching between the rods and the metallic block. In order to achieve the results shown in Fig. 7, other parameters were adjusted along with the syntonizing capacitance $C$, namely the lengths and radius of the rods. Increasing the radius $r$ of the rods contributes positively to reduce the return loss values (dotted lines in Fig. 7(a)). The highest value used for the radius of the rod, which provides precision of the simulations and minimizes the return loss, is $12.65 \mathrm{~mm}$.

The antenna used in the experiments is $15.6 \mathrm{~mm}$ in length (Fig. 3 (a)). However, the spatial step of the FDTD lattice was set to $3 \mathrm{~cm}$ due to computational restrictions imposed by the indoor environment. Thus, the equivalent model has a length of $21 \mathrm{~cm}$ as shown by Fig. 3 (c) and, for this reason, the capacitors are also necessary for reducing the electrical length of the model, as it is illustrated in Fig. 7(a).

The solid lines in Fig.7(a) show that the return losses obtained with the capacitors of $0.5 \mu \mathrm{F}$ are closer to experimental curve of Fig. 5 for $1.05 \mathrm{GHz} \leq f \leq 1.30 \mathrm{GHz}$ than the return losses calculated for the preliminary dipole antenna (with no capacitors). For this reason, the value of the capacitance used in simulations is $C=0.5 \mu \mathrm{F}$. 


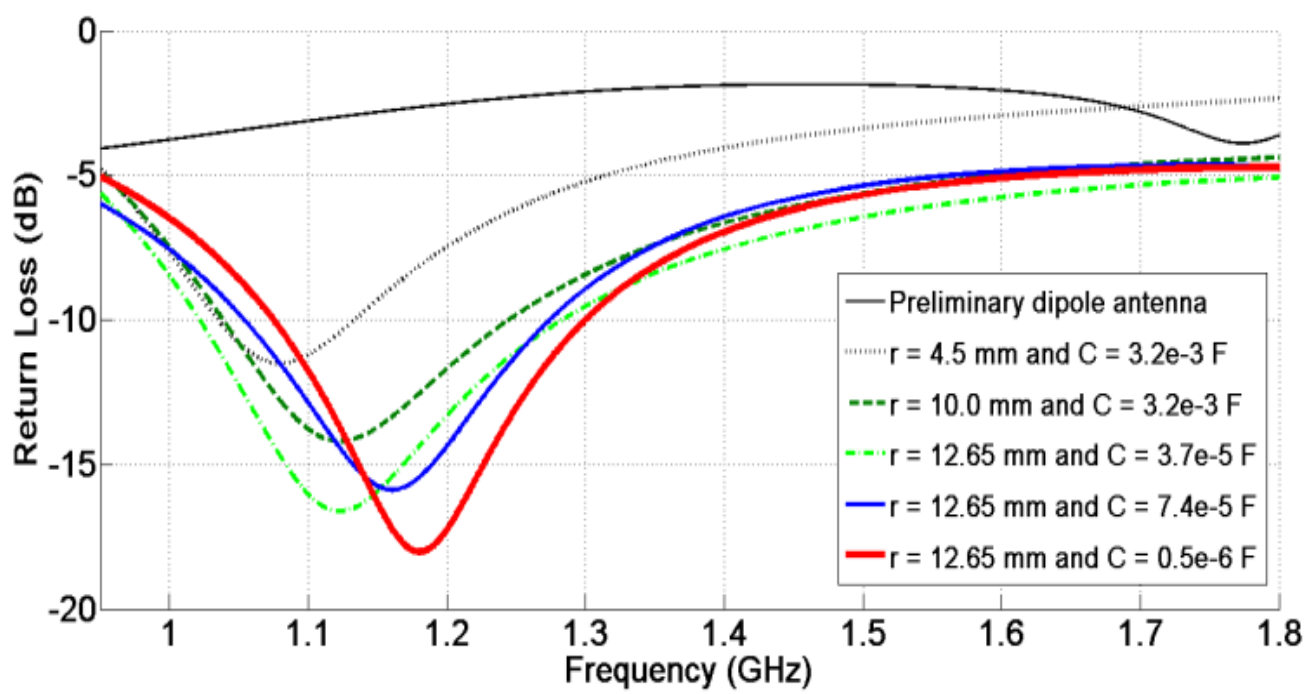

(a)

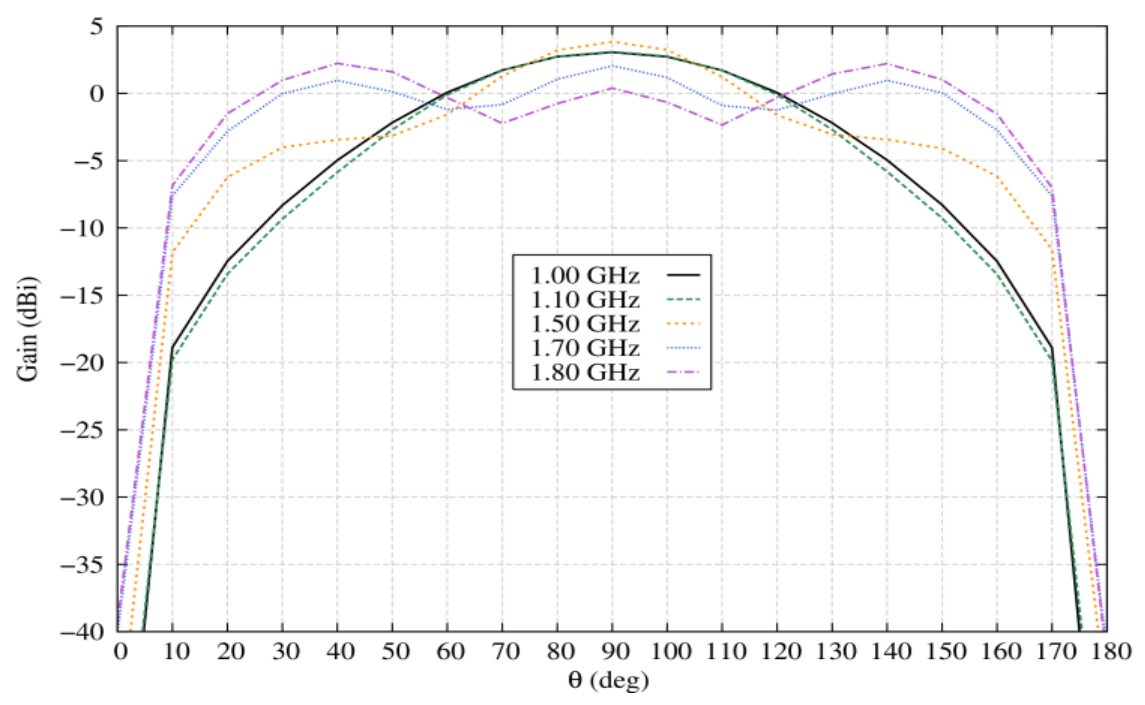

(b)

Fig. 7. Parameters of proposed antenna: (a) return losses for various capacitances and (b) gain obtained with capacitors of $0.5 \mu \mathrm{F}$.

The modeled antenna provides return losses of less than $-10 \mathrm{~dB}$, for approximately $35 \%$ of the frequency range of interest, as it can be seen in Fig. 7(a). For broadband antennas, it is suggested in [9] to consider the value of $-6 \mathrm{~dB}$ as a threshold in the study of return loss in the frequency range considered. The suggested antenna has $83 \%$ return loss below $-6 \mathrm{~dB}$. In addition, the entire return loss profile is under $-4 \mathrm{~dB}$ for the band of interest [12][13].

Finally, by comparing Figs. 4 and 7(b), it can be seen that the gain obtained using syntonizing capacitors of $0.5 \mu \mathrm{F}$ for the proposed antennas agree well with those obtained for the discone antenna in [3]. We see that for $1 \mathrm{GHz}$ and $1.1 \mathrm{GHz}$, the approximately omnidirectional profile is preserved by the equivalent model. Also, for 1.5, 1.7 and $1.8 \mathrm{GHz}$, the oscillations seen in gain profiles of Fig. 4 are represented reasonably well by the equivalent model, as shown by Fig.7 (b). 


\section{SIMULATION AND EXPERIMENTAL RESULTS}

For simulations of the environment depicted by Fig. 2 with the 3D-FDTD method, more than 32 GB of RAM were necessary. The used computer system is a Beowulf cluster [15] based on a distributed parallel architecture and on the message passing interface specification (LAM/MPI Library). The technical specifications of each of the 16 cluster nodes are: processor Intel Core i5-3330 working at $3.0 \mathrm{GHz}$ with $8 \mathrm{~GB}$ of RAM. The physical communication between the computers is performed via a 16-port-switch with transfer rate of $1000 \mathrm{Mbps}$. The operating system used is the Slackware Linux 64 bits, release 14.0. The processing time of 3D-FDTD simulation was approximately six hours. An exact antenna representation has not been considered in this paper for FDTD simulations. One of the motives is that the antenna is electrically small. Numerically, locally non-orthogonal or locally refined orthogonal grids $[2,14]$ could be used for representing the antenna. However, the use of non-uniform FDTD lattices with very small cells would require a much larger simulation time than the processing time necessary to conclude the FDTD simulation with the equivalent antenna representation proposed in this work. This is mainly due to the mandatory significant reduction of time step [2].

In order to validate the proposed FDTD equivalent antenna representation, and because it is not possible to resolve problems modeled with 3D-FDTD without using a radiator as well as it is not feasible to model an isotropic source [2], we used the polyhedral beam-tracing method (PBT), which was previously validated in [3] with excitation by an isotropic radiator. PBT is a frequency domain method, which is described in [3,9]. The PBT model ran on a notebook equipped with a $2.0 \mathrm{GHz}$ Intel Core 2 Duo processor with 1.0 GB of RAM. The Windows 7.0 operating system was employed. The processing time was 44.2 and 42.1 minutes for simulations with non-isotropic and isotropic antennas, respectively. The higher FDTD processing time compared to PBT is expected since in FDTD one needs to discretize the entire environment using electrically small Yee cells. In addition, all the six field components are calculated in each Yee cell for every discrete advance in time.

\section{A. Analysis of the measured data}

According to the configuration of the measurement campaign described in Section II, amplitudes and phases of the signals were obtained for several points in the frequency band of interest (950$1800 \mathrm{MHz})$. These values are used to calculate the power delay profile [1] at the transceiver positions (Fig. 2). The power delay profile measured at the first position (receiver Rx positioned one meter away from the transmitter Tx) is shown in Fig. 8 and displays several reflection peaks. These peaks can provide locations of specific objects in scenario. Locations can be determined by estimating the time that the signal needs to propagate from the transmitter to a reflecting object and finally arrive at the receiving antenna. 


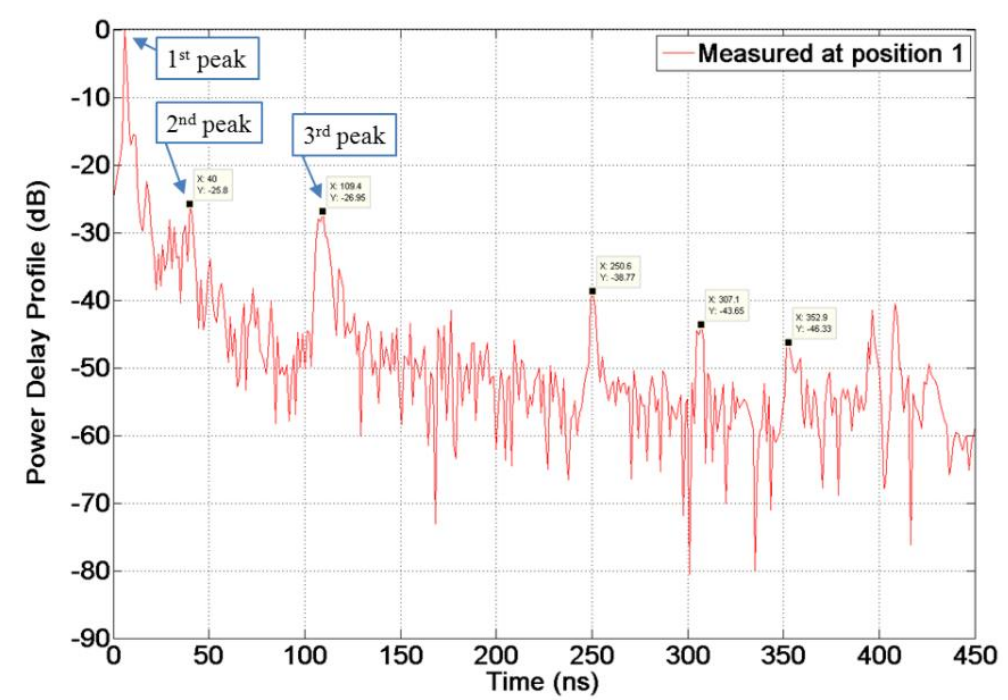

Fig. 8. Power delay profile measured at Tx1. Transmitter is 1 meter away from receiver.

Therefore, one can relate the time at which a peak is observed to walls and other objects in the environment shown in Fig. 2. In Fig. 8, the first peak is due to direct wave incidence (the signal propagates directly from the transmitter to the receiver). The second peak arrival time at the receiver was observed to be $40 \mathrm{~ns}$. This time defines a total propagating distance of $11.96 \mathrm{~m}$. This distance is likely to be associated with signal reflected in the stairs which are on the left side of the receiver (Fig.2). Finally, in Table III we have estimated associations of the other peaks of the signal shown in Fig. 8 with the scattering objects in the environment.

TABLE III ESTIMATED ASSOCIATIONS BETWEEN PEAK TIMES AND DISTANCES TO SCATTERING OBJECTS

\begin{tabular}{ccl}
\hline Peak Time (ns) & $\begin{array}{c}\text { Total Propagation } \\
\text { Path }(\mathbf{m})\end{array}$ & \multicolumn{1}{c}{ Object } \\
\hline 17.65 & 5.28 & Corridor wall \\
40.00 & 11.96 & Stairs 1 \\
109.4 & 32.7 & Wall with glass door \\
250.6 & 74.93 & Cabinet 1 \\
307.1 & 91.82 & Cabinet 2 \\
352.9 & 105.52 & Cabinet 3 \\
\hline
\end{tabular}

\section{B. Simulation results}

In order to obtain the time domain power delay profile from transient FDTD signals, it is necessary to implement a frequency-domain deconvolution algorithm to process the received signal. Specifically, calculations are partially performed in frequency domain (using Fourier transforms) for determining the channel transfer function $H(f)$ from the time domain voltages obtained between the terminals of the transmitter (Fig.3) and between the terminals of the receiving antenna. The voltages were calculated across the respective antennas' gaps.

The propagation channel is considered to be purely linear. It can be thought as a linear system with the transfer function $H(f)$. Consequently, the Fourier transform $V_{i}(f)$ of the transmitter voltage shown by Fig. 6(b) is the input of the linear system. The output signal $V_{\text {ox }}(f)$ is the Fourier transform of the transient voltage obtained between the terminals of a given receiver RX. Therefore, $H(f)=V_{\text {ox }}(f) / V_{\mathrm{i}}(f)$. 
The transient input and output voltages, obtained via FDTD method, are converted to the frequency domain using the Filon integration method [16]. The discrete spectral values of the voltages thus obtained are point-by-point divided for obtaining a discrete version of the channel transfer function $H(f)$. Afterwards, one proceeds with the calculation of the impulse response $h(\tau)$ of the channel using $h(\tau)=\mathcal{F}^{-1}\{H(f)\}$, where $\mathcal{F}^{-1}$ indicates the discrete inverse Fourier transform. The power delay profile is defined in decibels as $P D P=20 \log _{10}|h(\tau)|$.

Since the return loss is a measure of the quantity of power returning to the source, we can say that, with an adequate interpretation, it can indicate the amount of the power radiated by the antenna in a given frequency (considering Joule effect negligible). In addition, because the indoor environment can be considered as a linear system, from the physical point of view, it is easy to see that the amount of power radiated by the antenna for a given frequency is, in fact, not important for obtaining $H(f)$ and consequently not important to define PDP. However, from the numerical point of view, if the amplitudes of the fields at a given frequency are too low at the receiver, rounding errors on numerical evaluations can produce incorrect values for $H(f)$. For this reason, the return loss of the equivalent transmitting antenna must be under $-4 \mathrm{~dB}$ for the entire band of interest for ensuring that the signal reaches the receiver with appreciable amplitudes in order to avoid rounding problems. This also means that obtaining a specific profile of the return loss is not essential because $H(f)$ is a relative quantity.

For the point $\mathrm{Tx}_{1}$ in Fig. 2, the results obtained using the 3D-FDTD and PBT methods are compared with the experimental curve for power delay profile in Fig. 9. It can be seen in this figure that when the proposed antenna model is used in the receiver at $\mathrm{Rx}_{1}$, the FDTD method can reproduce most of the peaks shown in Fig. 8. The layout of the environment directly affects the amplitude and position of the peaks obtained in the simulation. When the receiving antenna was removed, certain peaks were inappropriately amplified (e.g. between 50 and $100 \mathrm{~ns}$ ) and other were also improperly attenuated (e.g. in the interval between 275 and $300 \mathrm{~ns}$ ). This is strongly related to the radiation pattern of the modeled receiving antenna, which is similar to the radiation profile of the discone antenna, as one can see in Figs. 4 and 7(b). For the PBT results without antenna (Fig. 9b), we see that some peaks are reproduced properly. However, for $t>50 \mathrm{~ns}$, power delay profile is below the experimental and FDTD curves. The average amplitude displacement is approximately $-10 \mathrm{~dB}$. This is explained by the fact that the transmitting and receiving antennas were not considered in the present PBT model simulation, differently from that performed in [3]. Consequently, each ray that goes out from the transmitter in different directions (or arriving at the receiver) endures a 0dbi gain for all frequencies and directions. When the transmitting antenna is absent, PBT results are equivalent to those produced by using an isotropic radiator. However, when the antennas gain is considered in PBT (Figs. 9b and 9c), the PDP amplitudes behave as if they were the moving average values of the measured PDP amplitudes. Notice that the numerical results in Fig. 9 that best fit the experimental curve is obtained with 3D-FDTD models in which the transmitter and receiver antennas are 
simultaneously included. Observe that performing 3D-FDTD simulations without antennas is not possible [2]. It is worth to notice that for Fig. 10, in which results are obtained $44.5 \mathrm{~m}$ away from the transmitter, PBT results present deviations similar to those seen in Fig. 9 (acquired $1 \mathrm{~m}$ from transmitter).

For 3D-FDTD simulation, the multipath amplitudes in Figs. 9 and 10 present similar oscillating behavior as in the measurement, especially for the delay values higher than $150 \mathrm{~ns}$. The PBT power delay profiles is relatively smooth, in comparison with the corresponding FDTD PDPs, since the PBT model consider up to 11 interactions (reflections or transmission) with the environment [3]. Therefore, multipath amplitudes were not detected due to the limited rays interaction with the environment's objects.

The simulated and measured parameters of the temporal fading are shown in Table IV for distances 1.0, 24.0 and 44.5 meters between transmitter and receiver. By analyzing PBT results, we see that the inclusion of the antenna radiation characteristics improve results over PBT calculations which are performed by using the isotropic radiator. However, numerical solutions obtained with 3D-FDTD employing the proposed equivalent antenna representation are in much better accordance to the measured data, validating the proposed methodology. Although a small part of the peaks are not present in FDTD results, the arrival time of significant multipath components are very similar to the experimental signal. The temporal fading of the channel was obtained considering the threshold of $50 \mathrm{~dB}$ for Excess Delay Spread.

TABLE IV TIME SPREAD OF THE CHANNEL

\begin{tabular}{l|ccc|ccc}
\hline \multirow{2}{*}{ Method } & \multicolumn{3}{|c|}{ Mean Excess Delay $-\overline{\boldsymbol{\tau}}(\mathbf{n s})$} & \multicolumn{3}{c}{ RMS Delay Spread $\boldsymbol{\tau}_{\boldsymbol{r m s}}(\mathbf{n s})$} \\
\hline Distance & $\mathbf{1 m}$ & $\mathbf{2 4} \mathbf{~ m}$ & $\mathbf{4 4 . 5} \mathbf{~ m}$ & $\mathbf{1 m}$ & $\mathbf{2 4} \mathbf{~ m}$ & $\mathbf{4 4 . 5} \mathbf{~ m}$ \\
\hline Measured & 7.934 & 133.333 & 210.560 & 12.351 & 57.798 & 55.823 \\
3D-FDTD & 8.604 & 125.170 & 201.290 & 14.783 & 54.869 & 60.392 \\
PBT (isotropic radiator) & 6.904 & 123.190 & 192.130 & 8.0492 & 46.357 & 46.721 \\
PBT (non-isotropic antenna) & 7.402 & 119.680 & 197.280 & 11.714 & 45.419 & 48.686 \\
\hline
\end{tabular}

PBT results in Figs. 9 and 10 lead to interesting conclusions regarding the impacts of the shape of the equivalent antenna used in FDTD method. With PBT, we can model an isotropic source [9]. Of course, radiation level is considered to be identical in every direction and for every frequency considered (specifically, electric field is considered to always have unitary amplitude). The transmitter is transparent: when electromagnetic field reflects in an object and returns to the source, the field unimpededly goes through it. For the case in which a more realistic antenna is considered in PBT, radiation levels are corrected for each direction and for each frequency. However, the antenna itself is also considered to be transparent [9]. In these PBT cases, even when the transparent antenna model is employed (which is evidently not the case of experiments), we see in Figs. 9 and 10 and in [9] that most of the prominent peaks of PDP are correctly predicted. It is also easy to see that most of 
the reflected signals (in the transmitting antenna) are of small amplitudes and thus have influence mainly on low amplitude portions of the PDP signals. This means that radiation pattern is of more importance than considering the exact shape of the antenna. This explanation justifies the fact that FDTD results, obtained with the proposed equivalent antenna model, are in very good accordance with experimental results: the radiation characteristics obtained with the model are compatible with those obtained experimentally (Fig. 7). As previously discussed and predicted, radiation characteristics are more important than representing in details the shape of the antenna for this kind of problem. The equivalent antenna model cannot be electrically very large, otherwise reflections can be appreciable and substantially modify the results and radiation characteristics can be drastically affected.

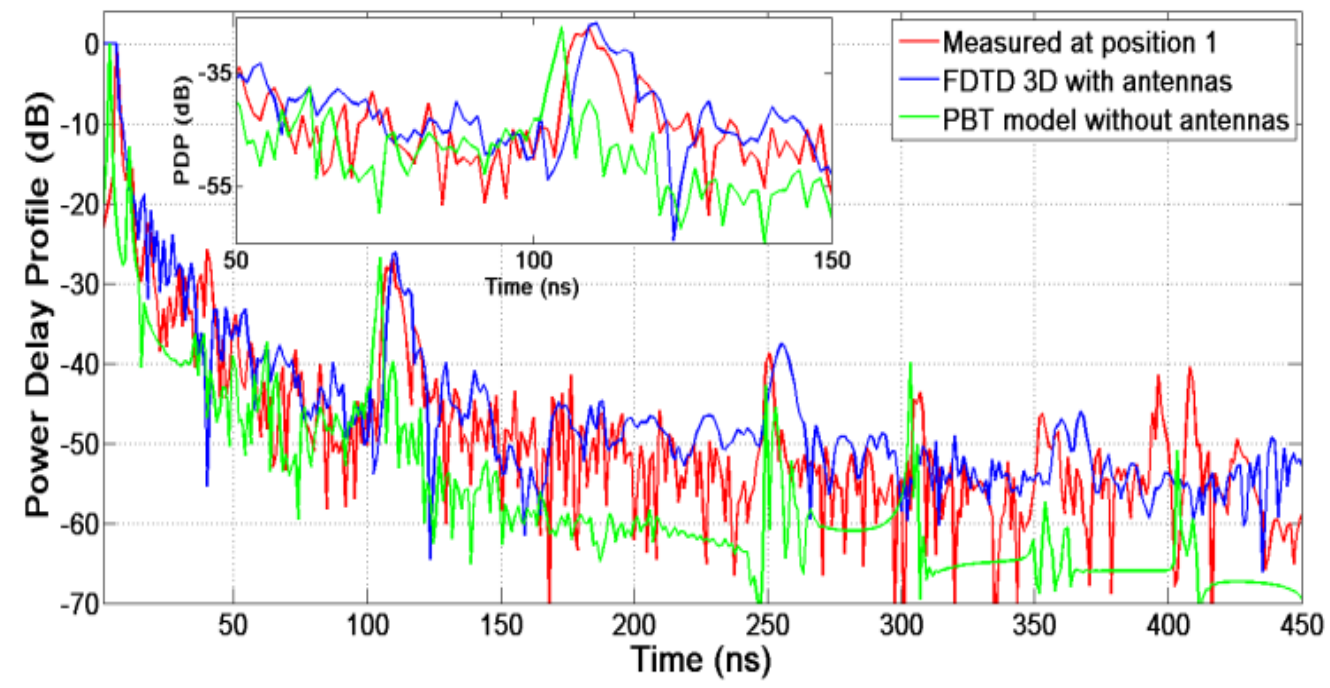

(a)

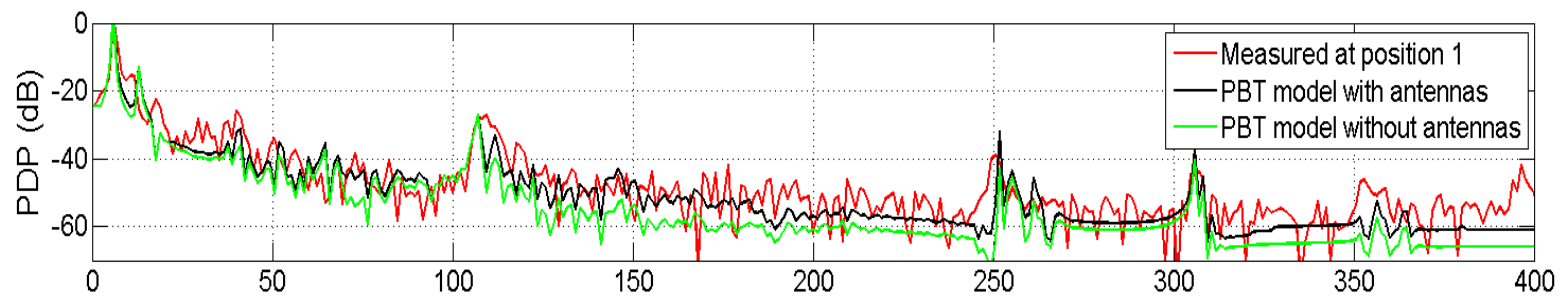

(b)

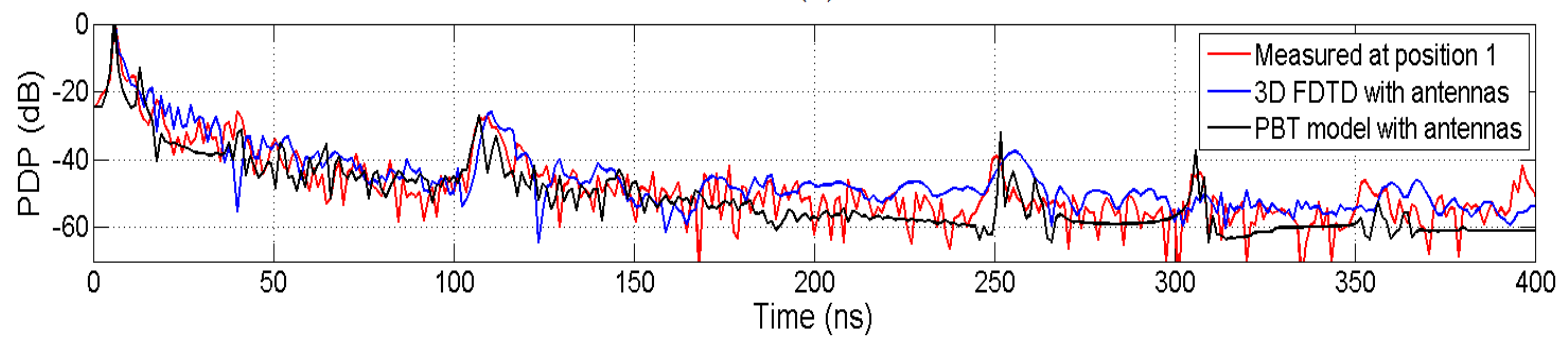

(c)

Fig. 9. Normalized PDP - transmitter is placed $1 \mathrm{~m}$ away from receiver: (a) experimental, FDTD and PBT results with isotropic radiator (insert: PDP for time period between 50 and $150 \mathrm{~ns}$ ), (b) experimental and PBT results with isotropic and non-isotropic radiators and (c) experimental, FDTD and PBT results with non-isotropic radiator. 


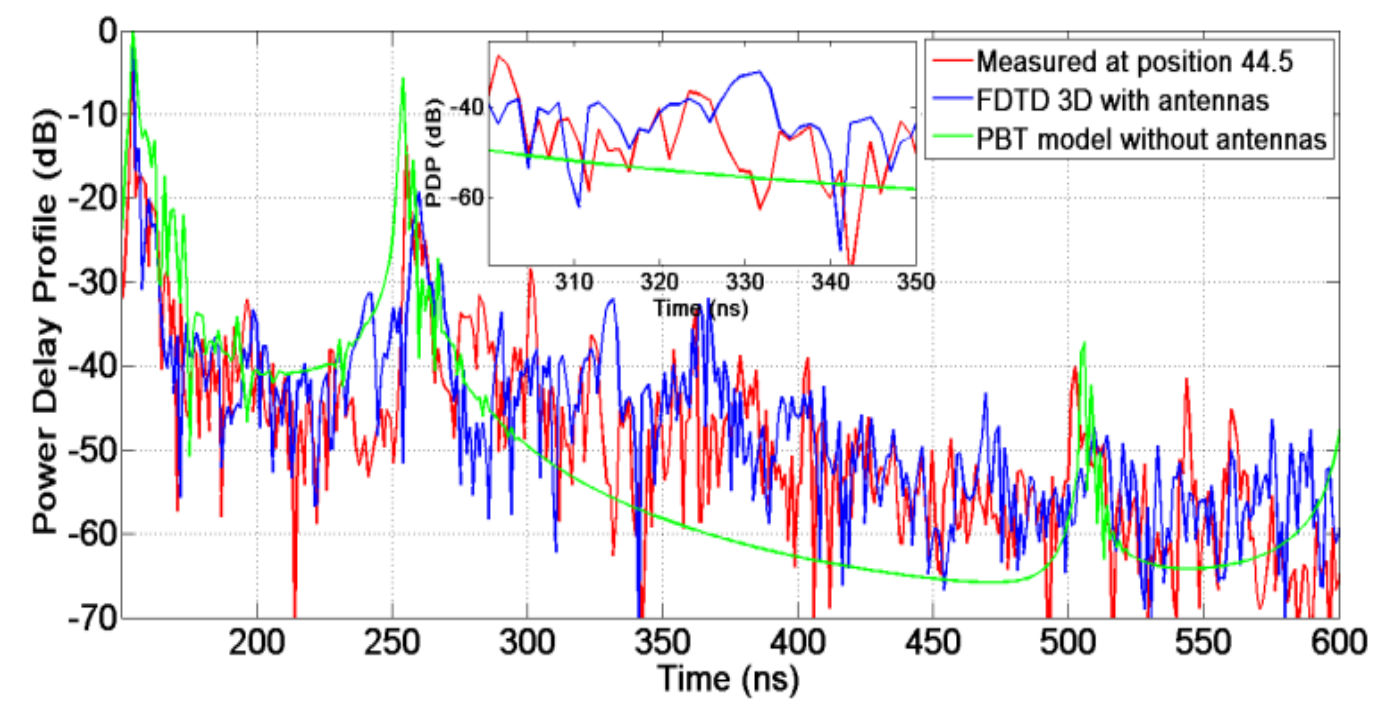

(a)

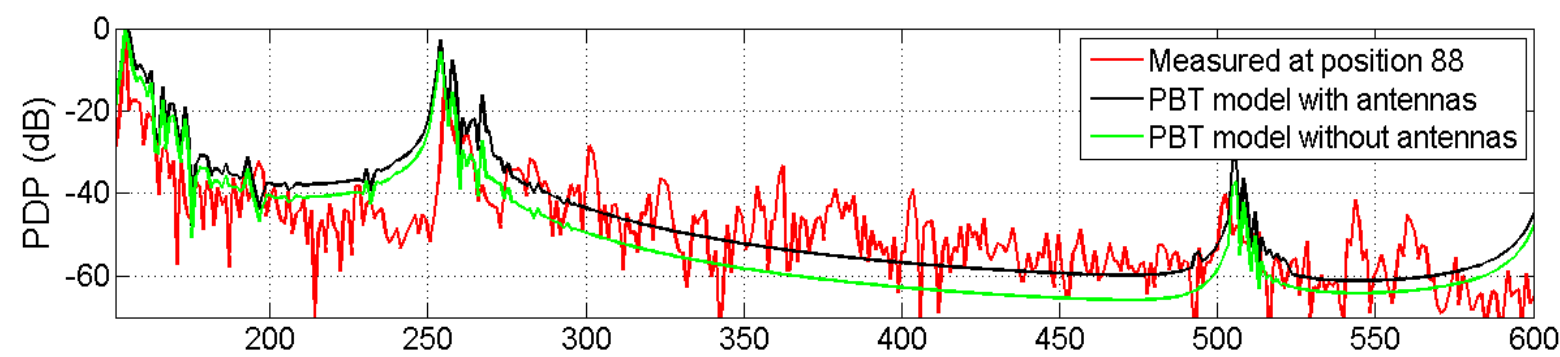

(b)

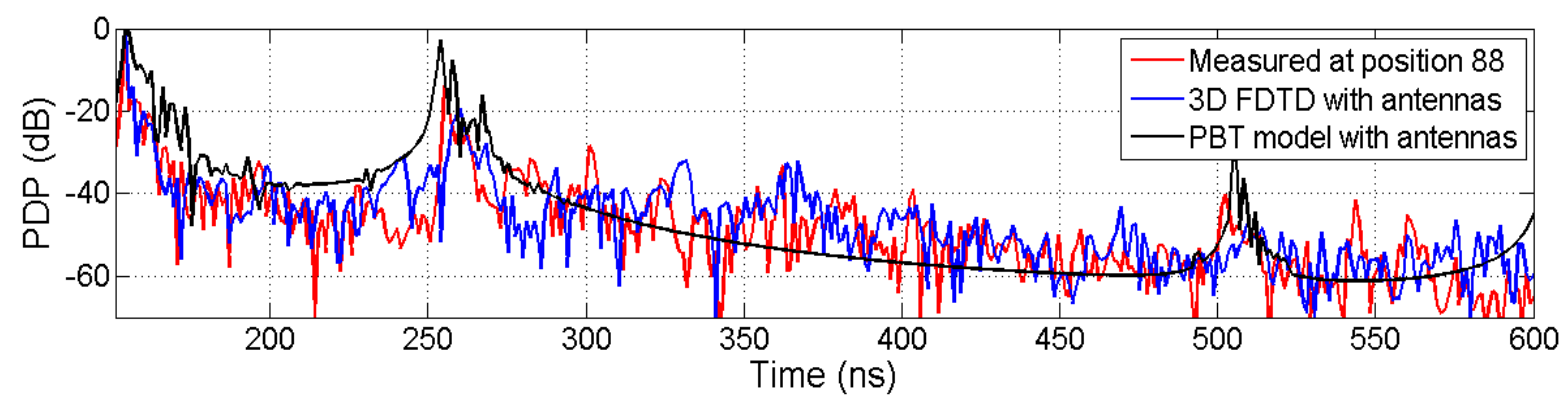

(c)

Fig.10. Normalized PDP - transmitter is placed $44.5 \mathrm{~m}$ away from receiver: (a) experimental, FDTD and PBT results with isotropic radiator (insert: PDP for time period between 300 and $350 \mathrm{~ns}$ ), (b) experimental and PBT results with isotropic and non-isotropic radiators and (c) experimental, FDTD and PBT results with non-isotropic radiator.

\section{CONCLUSIONS}

In this paper, indoor power delay profile is calculated using the classic FDTD method. In order to minimize processing time and the computational requirements for simulating the electrically large indoor environment analyzed, a coarse uniform FDTD grid is employed. Experiments were performed using a discone antenna. This antenna has electrically small geometric features, which cannot be represented with the coarse FDTD lattice. It is shown that, for this kind of numerical analysis, representing these features it is not required. The most important aspect is to replicate the radiation 
characteristics of the device used in experiments. In this context, an equivalent model is proposed for the discone antenna. Numerical tests performed with the suggested representation were exceptionally successful: FDTD results are in very good accordance with experiments, validating the equivalent FDTD model developed for the discone antenna.

\section{ACKNOWLEDGMENTS}

This work was partially supported by CAPES via the RH-TV Digital project. Authors acknowledge the support received from researchers of PUC-Rio, especially professors Glaucio L. Siqueira, José R. Bergmann and Emanoel Costa. The first researcher, professor Siqueira, the master's degree advisor of Fabrício Barros (the fourth author of the paper), provided valuable support for executing the experimental measurements. Professor Bergmann also provided important insights for performing measurements. Finally, prof. Costa (PhD advisor of F. Barros) granted relevant technical discussions necessary for development of the PBT model.

\section{REFERENCES}

[1] H. Hashemi, "The Indoor Radio Propagation Channel”, Proceedings of the IEEE, vol. 81, pp. 943-968, 1993.

[2] A. Taflove and S. C. Hagness, Computational Electrodynamics, The Finite-Difference Time-Domain Method. Artech House Inc., 2005.

[3] F. J. B. Barros, E. Costa, G. L. Siqueira and J. R. Bergmann, "A polyhedral beam-tracing method for modeling ultrawideband indoor radio propagation”, Microwave and Optical Technology Letters, vol.54, pp. 904-909, 2012.

[4] J. R. Bergman, On the design of broad band omnidirectional compact antennas. IEEE Microwave and Optical Technology Letters, vol. 39, pp. 418-422, October 2003.

[5] F. J. B. Barros, R. D. Vieira and G. L. Siqueira, "Relationship between delay spread and coherence bandwidth for UWB transmission," SBMO/IEEE MTT-S International Conference on Microwave and Optoelectronics, pp. 415-420, 2005.

[6] U. S. Inan and R. A. Marshall, Numerical Electromagnetics: the FDTD Method. Cambridge University Press, 2011.

[7] R. M. S. de Oliveira, and C. Sobrinho, "UPML Formulation for Truncating Conductive Media in Curvilinear Coordinates", Numerical Algorithms, vol. 46, pp. 295-319, 2007.

[8] R. M. S. de Oliveira and C. Sobrinho, "Computational environment for simulating lightning strokes in a power substation by finite-difference time-domain method", IEEE Transactions on Electromagnetic Compatibility, vol. 51, pp. 995-1000, 2009.

[9] F. J. B. Barros, E. Costa, G. L. Siqueira and J. R. Bergmann, "A Site-Specific Beam Tracing Model of the UWB Indoor Radio Propagation Channel”, IEEE Transactions on Antennas and Propagation, vol. 63,pp.3681-3694, 2015.

[10] H. Karl and A. Willig, Protocols and Architectures for Wireless Sensor Networks, John Wiley \& Sons, 2007.

[11] T. K. Sarkar, M. C. Wicks, M. S.-Palma and R. J. Bonneau, "Smart Antennas”, John Wiley \& Sons, 2003.

[12] I. Henning, M. Adams, Y. Sun, D. Moodie, D. Rogers, P. Cannard, S. Dosanjh, M. Skuse, and R. Firth, "Broadband antenna-integrated, edge-coupled photomixers for tuneable terahertz sources," IEEE Journal of Quantum Electronics, vol. 46, pp. 1498-1505, 2010.

[13] Z. Chen, Y. L. Ban, J. H. Chen, J. L. W. Li and Y. J. Wu, "Bandwidth Enhancement of LTE/WWAN Printed Mobile Phone Antenna Using Slotted Ground Structure". Progress In Electromagnetics Research, vol. 129, pp. 469-483, 2012.

[14] T. Noda, A. Tatematsu and S. Yokoyama, Improvements of an FDTD-Based Surge Simulation Code and its Application to the Lightning Overvoltage Calculation of a Transmission Tower, Electric Power Systems Research, vol. 77, pp. 1495-1500, 2007.

[15] D. J. Becker, T. Sterling, D. Savarese, J. E. Dorband, U. A. Ranawake and C. V. Packer, "BEOWULF: A parallel workstation for scientific computation", in Proceedings of International Conference on Parallel Processing, vol. 95, pp. $1-4,1995$.

[16] G. V. Milovanovic and M. Rassias, Analytic Number Theory, Approximation Theory and Special Functions, Springer, 2014. 